\title{
Distribuição espacial do risco de feminicídio em Campinas - São Paulo, Brasil
}

\author{
Spatial distribution of the risk of femicide in Campinas - São Paulo, Brazil \\ Distribución espacial del riesgo de feminicidio en Campinas - São Paulo, Brasil
}

Recebido: 21/12/2021 | Revisado: 28/12/2021 | Aceito: 03/01/2022 | Publicado: 04/01/2022

\author{
Mônica Caicedo-Roa \\ ORCID: https://orcid.org/0000-0001-7313-8260 \\ Universidade Estadual de Campinas, Brasil \\ E-mail: monicacaicedoroa@gmail.com \\ Ricardo Carlos Cordeiro \\ ORCID: https://orcid.org/0000-0002-0437-1066 \\ Universidade Estadual de Campinas, Brasil \\ E-mail: rica@unicamp.br \\ Lourdes Maria Bandeira \\ ORCID: https://orcid.org/0000-0002-7666-5338 \\ Universidade de Brasília, Brasil \\ E-mail: lourdesmbandeira@yahoo.com.br
}

\begin{abstract}
Resumo
Os feminicídios não se distribuem de forma aleatória, são produto de uma convergência de fatores sociais políticos, econômicos e culturais que confluem para que aconteçam eventos violentos no território. O objetivo do presente estudo é analisar a distribuição espacial do risco de morte por feminicídio na cidade de Campinas, São Paulo - Brasil por meio de um estudo caso-controle. Pesquisadores treinados realizaram a coleta das informações dos casos utilizando a técnica da autopsia verbal. Durante os anos 2018 e 2019 aconteceram 24 casos de feminicídio. Mulheres vítimas faleceram principalmente em locais residências em consequência de ataques com objetos perfurocortantes proporcionados por conhecidos principalmente namorados e conjugues. Os casos foram distribuídos no território e emparelhados com moradoras da cidade em uma relação de 1:4. Foi desenvolvida uma análise espacial identificando os locais de moradia e morte das mulheres vítimas. Por meio de um modelo linear generalizado se determinaram as regiões noroeste e sudoeste da cidade como aquelas nas quais existe maior risco de ocorrência de casos. São discutidos os achados no marco da normatividade legal brasileira e os eixos de intervenção para a eliminação da violência letal contra as mulheres no contexto Latino-americano.
\end{abstract}

Palavras-chave: Violência por parceiro Íntimo; Violência contra a mulher; Violência de gênero; Feminicídio; Análise espacial.

\begin{abstract}
Feminicides are not distributed randomly, they are the product of a convergence of social, political, economic and cultural factors that come together to cause violent events to happen in the territory. The aim of the present study is to analyze the spatial distribution of the risk of death from femicide in the city of Campinas, São Paulo - Brazil through a case-control study. Trained researchers collected information from the cases using the technique of verbal autopsy. During 2018 and 2019 there were 24 cases of femicide. Female victims died mainly in their homes as a result of attacks with sharp objects provided mainly boyfriends and spouses. Cases were distributed across the territory and paired with city controls in a 1:4 ratio. A spatial analysis was carried out identifying the places of residence and death of female victims. Using a generalized linear model, the northwest and southwest regions of the city were determined as those in which there is a greater risk of occurrence of cases. The findings are discussed within the framework of Brazilian legal normativity and the intervention axes for the elimination of lethal violence against women in the Latin American context.
\end{abstract}

Keywords: Intimate partner violence; Violence against women; Gender-based violence; Femicide; Spatial analysis.

\section{Resumen}

Los feminicidios no se distribuyen aleatoriamente, son producto de la convergencia de factores sociales, políticos, económicos y culturales que se unen para provocar hechos violentos en el territorio. El objetivo del presente estudio es analizar la distribución espacial del riesgo de muerte por femicidio en la ciudad de Campinas, São Paulo - Brasil a través de un estudio de casos y controles. Investigadores entrenados recopilaron información de los casos utilizando la técnica de autopsia verbal. Durante 2018 y 2019 hubo 24 casos de feminicidio. Las mujeres víctimas murieron principalmente en sus hogares como consecuencia de ataques con objetos punzantes proporcionados por conocidos, principalmente novios y cónyuges. Los casos se distribuyeron por todo el territorio y se emparejaron con los habitantes de la ciudad en una relación de 1: 4. Se realizó un análisis espacial identificando los lugares de residencia y muerte de las mujeres víctimas. Utilizando un modelo lineal generalizado, se determinaron las regiones noroeste y suroeste de la ciudad como 
aquellas en las que existe un mayor riesgo de ocurrencia de casos. Los hallazgos se discuten en el marco de la normatividad jurídica brasileña y los ejes de intervención para la eliminación de la violencia letal contra las mujeres en el contexto latinoamericano.

Palabras clave: Violencia de pareja; Violencia contra la mujer; Violencia de género; Feminicidio; Análisis espacial.

\section{Introdução}

As violências contra as mulheres não se distribuem no espaço de forma aleatória. Uma série de fatores confluem para gerar regiões do território com maior ou menor probabilidade de acontecimentos violentos. Aspectos como a história das cidades, questões culturais e sociais, assim como o sistema econômico têm uma grande influência na forma como sucedem os fenômenos violentos.

Um feminicídio é a forma de violência mais extrema contra uma mulher. Os feminicídios não são eventos isolados, repentinos nem inesperados, fazem parte de um processo contínuo de uso de violência e violência extrema (Instituto Patricia Galvão). São resultado de manifestações violentas enraizadas nas relações de poder desigual entre homens e mulheres. Ditas desigualdades são sustentadas por valores sociais, religiosos, econômicos e práticas culturais (Ellsberg et al., 2000).

A Corte Interamericana de Direitos Humanos define femicídio como: A morte de uma mulher por gênero (Sosa, 2017). A Organização das Nações Unidas o define como "O assassinato de mulheres e meninas devido ao seu gênero, que pode assumir a forma de: 1 o assassinato de mulheres como resultado de violência praticada pelo parceiro íntimo; 2 . a tortura e assassinato misógino de mulheres 3. assassinato de mulheres e meninas em nome da "honra"; 4. assassinato seletivo de mulheres e meninas no contexto de um conflito armado; 5 . assassinatos de mulheres relacionados com o dote; 6 . assassinato de mulheres e meninas por causa de sua orientação sexual e identidade de gênero; 7 . assassinato de mulheres e meninas aborígenes e indígenas por causa de seu gênero; 8. infanticídio feminino e feticídio de seleção com base no sexo; 9. mortes relacionadas à mutilação genital; 10. mortes por acusações de feitiçaria; e 11. outros femicídios relacionados a gangues, crime organizado, tráfico de drogas, tráfico de pessoas e proliferação de armas pequenas” (United Nations \& Economic and Social Council, 2013).

No mundo todo acontecem feminicídios. Em 2019 diariamente 137 mulheres foram mortas por um membro da própria família (United Nations Office on Drugs and Crime, 2019). A maioria dos assassinatos femininos são na esfera privada e têm como autores os parceiros ou familiares de sexo masculino. Do total dos assassinatos contra mulheres estimou-se que 58\% foram feminicídios que tiveram o parceiro íntimo ou outros familiares como agressores (United Nations Office on Drugs and Crime, 2019). Suspeita-se que os registros da quantidade de casos estão subestimados devido a que alguns crimes permanecem encobertos como casos de suicídio ou mortes acidentais.

O Brasil é um país com uma cultura altamente tolerante à violência o que determina um problema sério de violência contra as mulheres. No Atlas da violência de 2019 foi reportado o crescimento dos homicídios femininos no país. Estimaram-se 13 assassinatos de mulheres por dia. Chama a atenção a desigualdade racial, onde do total das mulheres assassinadas o 66\% correspondem a vítimas de cor negra (Cerqueira \& Bueno, 2019). A mortalidade no país é elevada, mas não é uniforme nas regiões do território (Meneghel \& Hirakata, 2011).

Algumas formas de violência aumentaram como consequência da mudança nos papéis tradicionais de homens e mulheres, mudança que tornou possível o ingresso delas no mercado de trabalho e permitiu sua maior independência econômica e reprodutiva sendo essa situação um potencial gerador de conflitos (Meneghel \& Hirakata, 2011). Movimentos sociais e ideológicos de pensamento feminista têm facilitado a mudança de ditos papeis. Fatores como o desemprego masculino assim como a separação por parte da mulher são considerados de risco para feminicídio, mas o principal fator de risco é a violência doméstica previa (Campbell et al., 2007). 
No mundo quase um terço das mulheres que estiveram em um relacionamento (30\%, IC 95\%=27,8-32,2\%) relatam que experimentaram alguma forma de violência física e/ou sexual proveniente do parceiro íntimo durante a vida (World Health Organization, 2013).

Em concordância com os achados internacionais, no Brasil, a maior incidência de feminicídio ocorreu entre pessoas que têm, ou tiveram, uma relação afetiva estável, seguido por namorados ou ex-namorados. As principais motivações dos crimes foram separação ou rompimento do relacionamento, atos de ciúmes/machismo e discussões banais (Núcleo de Gênero Ministerio Público do Estado de São Paulo, 2018). Os feminicídas praticam crimes todos os dias a todas horas principalmente na casa da própria vítima, a casa comum do casal ou a dos parentes da vítima (Núcleo de Gênero Ministerio Público do Estado de São Paulo, 2018). A vitimização das mulheres dentro de casa, longe dos olhos da sociedade, reforça o caráter privado dos crimes, ao mesmo tempo que os meios empregados sugerem a desvantagem física e a desproteção da vítima em relação ao agressor (Pasinato, 2016). O médio maiormente empregado para os ataques são as armas brancas e de fogo (Núcleo de Gênero Ministerio Público do Estado de São Paulo, 2018).

O Brasil é o maior e mais povoado país da América Latina, com uma população estimada de 213,3 milhões de habitantes (Instituto Brasileiro de Geografia e Estatística, 2021b). A cidade de Campinas é a terceira mais populosa do estado de São Paulo, com uma população estimada de 1.223.237 pessoas em 2021 (Instituto Brasileiro de Geografia e Estatística, 2021a). Considerando e recorte territorial da cidade de Campinas, o objetivo deste estudo é analisar a distribuição espacial do risco de morte por feminicídio por meio de um estudo de casos e controles.

\section{Metodologia}

O presente trabalho foi realizado como um subproduto do estudo "Distribuição espacial do risco de acidente do trabalho fatal em campinas-SP-Brasil: um estudo caso-controle espacial" com financiamento da Fundação de amparo à pesquisa do estado de São Paulo (Fapesp) no qual Laboratório de Análise Espacial de Dados Epidemiológicos (epiGeo), durante o ano 2019, coletou dados de mortes por causas externas mediante autópsias verbais.

O epiGeo está composto por docentes e estudantes da Faculdade de Ciências Médicas da Universidade Estadual de Campinas interessados em epidemiologia e análise espacial. Desenvolve trabalhos em três linhas de pesquisa: linha de epidemiologia das causas externas e saúde, trabalho e ambiente e epidemiologia das doenças infecciosas (Unicamp \& Faculdade de Ciências Médicas, 2020). O epiGeo tem realizado análises de distribuição espacial principalmente para doenças crónicas (Lora, 2010), agravos de saúde relacionados ao trabalho (Stephan, 2008) e doenças transmissíveis. E vem consolidado o método de autópsias verbais em acidentes de trabalho (Ricardo Cordeiro, 2018; Hennington et al., 2004), violência urbana (R. Cordeiro, 2018; Cordeiro et al., 2017) e feminicídios (Caicedo Roa et al., 2021; Caicedo-Roa et al., 2019).

\section{Local de coleta de dados}

Campinas é uma cidade com 260 anos de história. Está localizada a 98km da capital do estado, com uma área de 801 km2. A cidade está formada por quatro distritos: Joaquim Egídio, Sousas, Barão Geraldo e Nova Aparecida e cinco macrorregiões (Figura 1). É a terceira cidade mais populosa do estado de São Paulo, ficando atrás de Guarulhos e da capital paulista (Câmara Municipal de Campinas, 2015). 
Figura 1. Mapa com a divisão por grandes regiões da cidade de Campinas.

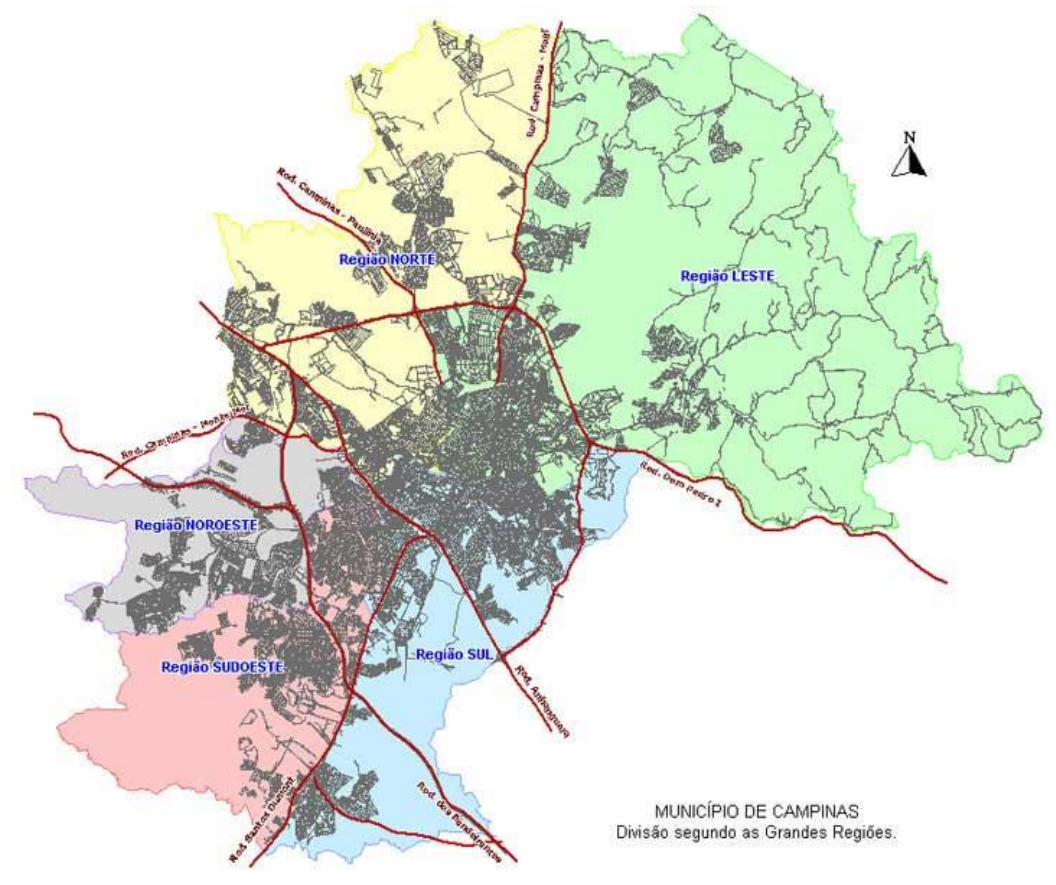

Fonte: Prefeitura de Campinas. http://www.campinas.sp.gov.br/governo/seplama/publicacoes/censo-2010.php

A cidade tem destaque no comércio, pesquisa, serviços de alta tecnologia e empresas na área de logística (Câmara Municipal de Campinas, 2015). Campinas como a maioria das cidades do território brasileiro apresenta um grande contraste socioeconômico. Existem regiões da cidade com grande infraestrutura e investimento e outras altamente precarizadas. A grosso modo em Campinas há uma macrorregião norte rica e uma macrorregião sul pobre (Cano \& Brandão, 2002). Estudos sobre a história da urbanização de Campinas no período de 1850-1908 têm mostrado a vinculação do desenvolvimento da cidade ao contraste entre o grande fluxo de capital pelas vastas plantações de cana de açúcar e café e a fome da população trabalhadora (Antipon, 2018).

Um fato importante para a urbanização e organização da cidade foram as epidemias de febre amarela de 1889, 1890, 1896 e 1897 que ao representar risco para os interesses dos proprietários e comerciantes cafeteiros, acabaram impulsionando a melhora das condições de vida da população mediante políticas públicas de saneamento e higiene. No entanto, a morte dos trabalhadores de fome e febre acabaram segregando a população mais pobre para as periferias da cidade (Antipon, 2018). Campinas passou por três períodos de periferização da população de baixa renda derivados da industrialização e modernização da cidade. O primeiro de 1940-1960, o segundo de 1970-1990 e o último desde 1990 até a atualidade (Rizzatti, 2015).

Atualmente Campinas, com todos seus contrastes está na $11^{\mathrm{a}}$ posição das cidades mais ricas do Brasil, o município contribui com $1 \%$ de todo o Produto Interno Bruto do País. A cidade é responsável por $10 \%$ de toda a produção científica nacional, sendo o terceiro maior polo de pesquisa e desenvolvimento brasileiro (Câmara Municipal de Campinas, 2015).

\section{Desenho epidemiológico do estudo e coleta de dados}

Para o estudo do fenômeno da violência letal contra as mulheres foi desenvolvido um estudo casos e controles, descritivo, com abordagem quantitativa. Sendo os casos as vítimas de feminicídio e os controles mulheres moradoras vivas da cidade. Foram realizadas autopsias verbais, método indireto para estimar uma causa específica de mortalidade utilizada para a recolecção, codificação e sistematização de informações de mortalidade (World Health Organization, 2007). 
Casos: para a identificação dos casos, foram obtidas as declarações de óbito de moradoras da cidade que faleceram por homicídio (códigos CID10 desde X850 a Y357) durante os anos 2018 e 2019 mediante uma parceria colaborativa com a Secretaria de Saúde. Posterior a 15 dias da data de falecimento foram visitados os domicílios das vítimas para a realização da autópsia verbal. Foram realizadas as autopsias em formato digital por meio de um tablet fazendo uso de um questionário semiestruturado e obtidas as coordenadas de latitude (Leste-Oeste) e longitude (Norte-Sul) dos locais de moradia e de morte das vítimas. Semanalmente os pesquisadores discutiam os casos sinalando os aspectos relevantes das entrevistas. Foram feitas discussões grupais sobre a classificação dos casos e definidos os que se enquadravam como feminicídios.

Controles: para a seleção dos controles foi realizada uma amostragem aleatória nos domicílios da cidade de Campinas fazendo uso do cadastro de domicílios residenciais da Sociedade de Abastecimento de Água e Saneamento. Foi obtida uma amostra aleatória simples sem reposição de 800 domicílios, juntamente com suas coordenadas geográficas. Ditos domicílios, foram visitados pelos pesquisadores, mediante sorteio, foi escolhido um morador em cada domicílio e convidado a participar. Foram obtidas informações ocupacionais, demográficas e sociais. Do total de controles foi realizado um recorte por sexo empregando unicamente dados da população auto definida como feminina.

\section{Considerações éticas}

O estudo foi aprovado pelo Comitê de ética em pesquisa da Faculdade de Ciências Médicas da Unicamp, mediante os CAAE: 04005118.9.0000.5404 e 29654720.6.0000.5404. Os Termos de consentimento livre esclarecido foram solicitados para os participantes na sua condição de familiares de casos ou controles segundo correspondesse e foram garantidas as condições de confidencialidade e sigilo das informações coletadas.

\section{Distribuição espacial do risco e modelo linear generalizado}

A análise espacial é um recurso metodológico e analítico da epidemiologia. Esta técnica permite a identificação da distribuição dos fenômenos de interesse em saúde e sua possível associação com fatores econômicos, culturais, ambientais e sociais assim como o reconhecimento de grupos populacionais sob risco mediante a utilização de sistemas de informação georreferenciada (SIG). De forma muito sucinta a análise espacial permite o estudo da distribuição de fenômenos sociais e de saúde no espaço geográfico.

A análise espacial aplica três passos: visualização, exploração e modelagem. A visualização é o método mais amplamente utilizado para descrever padrões espaciais; a exploração espacial de dados inclui o uso de métodos estatísticos para determinar se os padrões observados se distribuem aleatoriamente no espaço e, a modelagem usa dados complementares aos espaciais para explicar ou predizer padrões espaciais (Pfeiffer et al., 2008).

Além de identificar a distribuição da ocorrência dos casos de interesse em um território a análise espacial permite verificar padrões ou tendências sugestivas de aglomeração de casos controlando por variáveis individuais. Esta análise permitem abordar simultaneamente aspectos do indivíduo e socioambientais, partindo da localização pontual da residência e tratando o espaço como uma superfície contínua (Carvalho \& Souza-Santos, 2005).

O método de distribuição espacial do risco se baseia na teoria de processos pontuais espaciais. Assim se define uma medida de risco que varia sobre a região que pode ser estimada por meio de modelos aditivos generalizados. A hipótese nula da análise espacial é que o risco se distribui uniformemente no território. Para a estimação do risco é de utilidade o grupo controle. O método oferece a possibilidade de avaliar simultaneamente a variação da distribuição espacial dos casos quando comparada com a distribuição espacial dos controles e definir contornos de tolerância que auxiliam na identificação de áreas onde o risco é significativamente maior ou menor à média global (Shimakura et al., 2001). 
Para compreender o modelo é necessário definir vários componentes:

- $S_{1}, S_{2}, S_{3}, \ldots, S_{n 1} \rightarrow$ correspondem com as localizações na região de estudo

- $A \rightarrow$ corresponde com a região de estudo

- $n_{1} \rightarrow$ casos

- $S_{n 1+1}, S_{n 1+2}, \ldots, S_{n} \rightarrow$ corresponde à localização dos $n-n_{1}$ controles

- Dos processos de Poisson I e II com intensidades $\lambda_{1}(\mathrm{~s})$ e $\lambda_{2}(\mathrm{~s})$

- O logaritmo do risco da localidade como $s$

$$
p(s)=\log \left\{\frac{\lambda_{1}(s)}{\lambda_{2}(s)}\right\}
$$

É objetivo da análise investigar a variação de $p(s)$ em $A$ (Shimakura et al., 2001). O presente trabalho utiliza uma abordagem semi-paramétrica com modelos aditivos generalizados. O modelo é semi-paramétrico porque tem uma parte paramétrica, que são as variáveis de exposição coletadas para casos e controles, e um componente não paramétrico que são os pontos no espaço identificados mediante coordenadas geográficas de latitude e longitude.

Considere-se novamente $S_{1}, S_{2}, S_{3}, \ldots, S_{n 1}$ as localizações dos $n_{1}$ casos e, $S_{n 1+1}, S_{n 1+2}, \ldots, S_{n}$ as localizações dos $n-$ $n_{1}$ controles. Seja $Y_{i}$ um indicador associado ao ponto $S_{i}$ tal que $Y_{i}=1$ se o indivíduo i for um caso ou 0 se for um controle.

Assume-se que $Y_{i}(i=1, \ldots, n)$ são realizações de variáveis aleatórias independentes $Y_{i} \sim \operatorname{Bernoulli}(\mathrm{p}(\mathrm{s}))$, onde:

$$
p(s)=\frac{q_{1} \lambda_{1}(s)}{q_{1} \lambda_{1}(s)+q_{2} \lambda_{2}(s)}
$$

- $\quad q_{1}$ representa a proporção de casos registrados

- $\quad q_{2}$ a proporção de controles registrados

Como regra se utiliza a transformação à escala logarítmica. Para a inclusão das co-variáveis se tem a formula:

$$
\log \left\{\frac{p(s, x)}{1-p(s, x)}\right\}=\beta \mathrm{x}+\mathrm{g}(\mathrm{s})
$$

Onde x é o vetor das covariáveis e $\beta$ sus efeitos e a única suposição de que $g$ e uma função suave, porém desconhecida das coordenadas espaciais $s$. Em particular, se o risco é assumido constante na região, então $\mathrm{g}(s)=0$. O modelo se traduz simplesmente em um modelo de regressão logística clássico estendido com uma componente aditiva g $(s)$ que varia suavemente no espaço. O procedimento de estimação de $\beta$ e $\mathrm{g}(s)$ se baseia em modelos iterativos de modelos aditivos generalizados (Shimakura et al., 2001).

$\mathrm{Na}$ análise espacial foram desenvolvidos mapas com a localização geográfica dos casos de feminicídio para identificar visualmente áreas de concentração de casos ou tendências espaciais e analisados em relação aos controles. As análises estatísticas e de distribuição espacial do risco foram executadas nos softwares estatísticos R (R Core Team, 2014) e Stata (StataCorp, 2011).

\section{Resultados}

Em total foram identificados 24 casos de feminicídio. As mulheres vítimas eram principalmente adultas entre os 26 e 50 anos, metade delas procedentes de fora do estado de São Paulo, eram trabalhadoras e expostas a violência doméstica. 
Faleceram principalmente em locais residências em consequência de ataques com objetos perfurocortantes. Na maioria dos casos os autores das violências eram conhecidos principalmente namorados e conjugues.

Os casos foram emparelhados com 96 mulheres controle, utilizando a relação de quatro controles para cada caso. O emparelhamento foi realizado considerando as faixas de idade (0-25 anos, 26-50 anos, 51-75 anos e >75 anos) e índice de desenvolvimento humano municipal (IDHM) (muito alto: 0.800/1.000, alto: 0.700/0.799, médio: 0.600/0.699, baixo: 0.500/0.599 e muto baixo: 0.000/0.499). O IDHM é uma medida composta de indicadores de três dimensões do desenvolvimento humano agrupados por meio da média geométrica: longevidade, educação e renda. O índice varia de 0 a 1 . Quanto mais próximo de 1, maior o desenvolvimento humano (Programa das Nações Unidas para o Desenvolvimento, 2020). Os IDHM foram assignados de forma individual a casos e controles segundo seu local de moradia. Na Figura 2 pode ser vista a localização da residência dos casos de feminicídio e dos controles.

Figura 2. Localização das moradias dos casos de feminicídio ( $\mathrm{n}=24)$ e as mulheres controle (n=96) da cidade de Campinas SP Brasil 2018-2019.

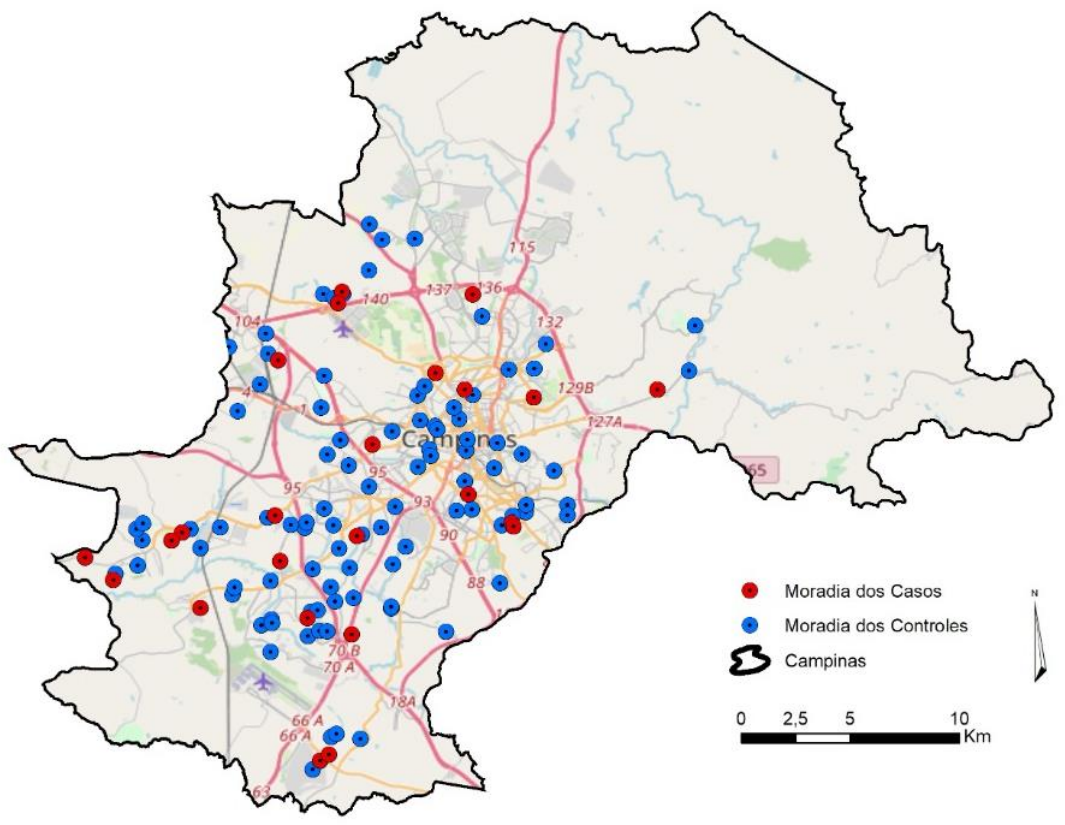

Fonte: Elaboração dos autores.

Na Figura 3 foram comparados os locais de moradia e de morte das mulheres vítimas de feminicidio. Pode se apreciar que na maioria dos casos os pontos se sobrepoem sendo o local de moradia o mesmo da morte. A respeito do total das mortes $71 \%$ acontreceram em uma residencia da vítima ou do agressor, $13 \%$ na rua, $13 \%$ em um terreno valdio e somente 1 caso (4\%) em um estabelecimento comercial. 
Figura 3. Locais de moradia (em vermelho) e morte (em preto) das 24 vitimas de feminicídio da cidade de Campinas- SP Brasil 2018-2019.

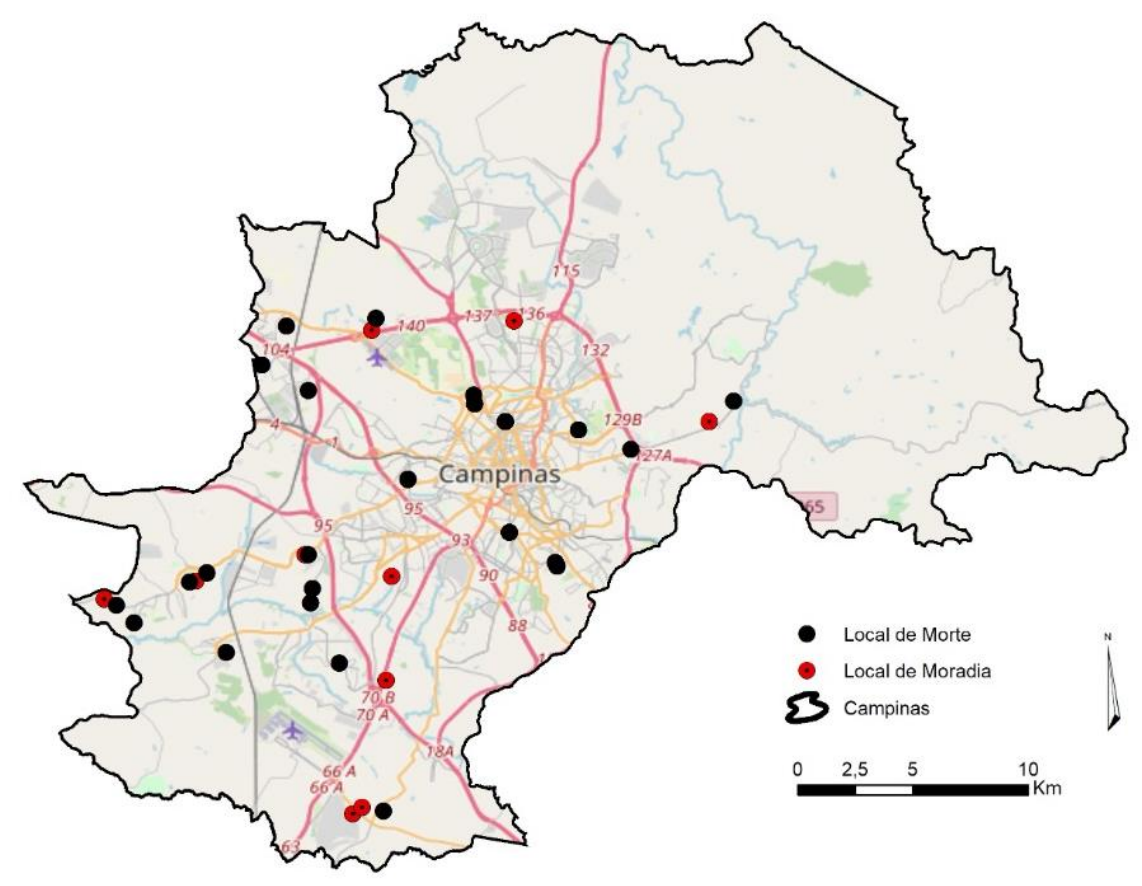

Fonte: Elaboração dos autores.

\section{Covariáveis do modelo linear generalizado}

Foi realizada uma análise de regressão logística binária condicional e identificadas duas variáveis do conjunto de dados que explicaram significativamente a probabilidade de ser um caso de feminicídio: a procedência fora do estado São Paulo e sofrer violência pelo parceiro íntimo nos últimos 30 dias. As duas, foram adicionadas como covariáveis no modelo linear generalizado junto com as coordenadas do local de moradia de casos e controles. Na Figura 4 pode ser apreciado o padrão de distribuição do risco de feminicídio na cidade de Campinas. 
Figura 4. Distribuição espacial do risco de feminicídios no território da cidade de Campinas. Os pontos se correspondem com a localização da moradia dos casos.

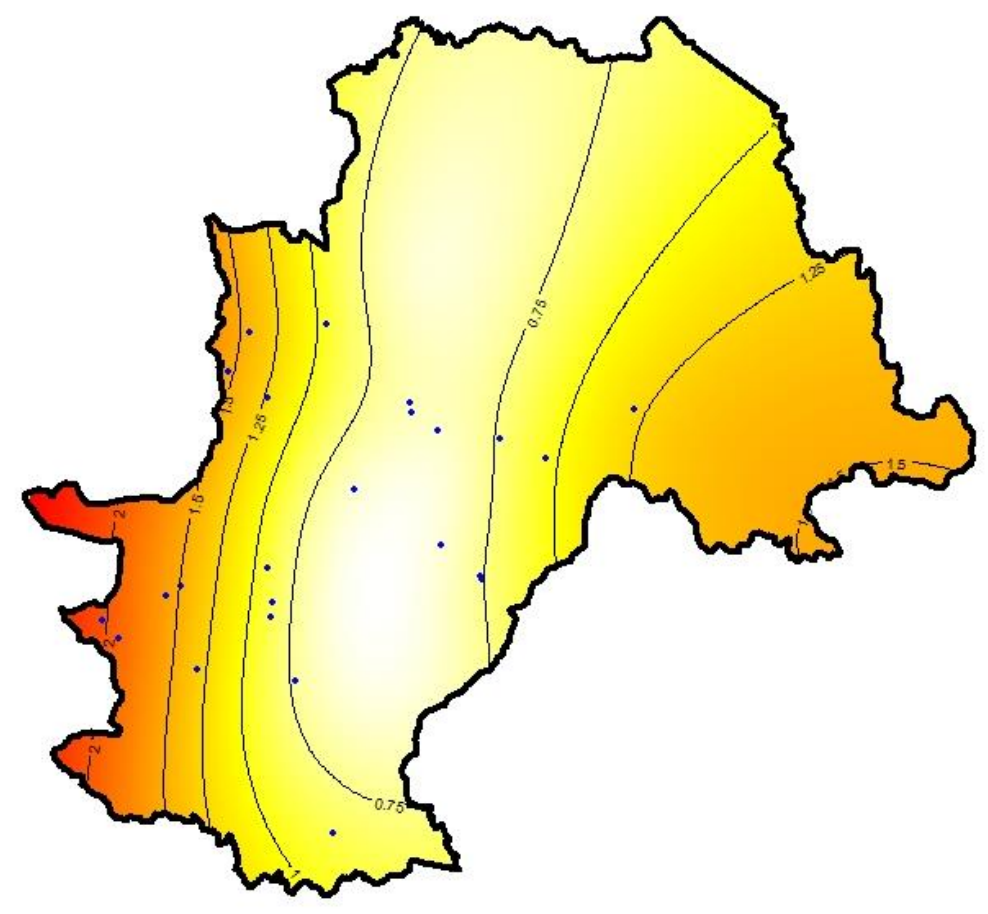

Fonte: Elaboração dos autores.

\section{Discussão}

Fazendo uso de uma análise espacial com um modelo linear generalizado foram localizados no território de Campinas os locais de moradia dos 24 casos de feminicídio que aconteceram nos anos 2018 e 2019 e comparados com um grupo de 96 mulheres controle. Desta forma, foram identificadas as regiões noroeste e sudoeste da cidade como aquelas nas quais existe maior risco de casos de feminicídio. Os casos aconteceram principalmente na região noroeste nos bairros Campina grande, Jardim Bassoli, Jardim Novo Maracanã, Jardim Nova Esperança e Dic V.

Em Campinas existem duas delegacias de defesa da mulher: uma localizada no Jardim Pauliceia e a outra no Jardim Proença I as duas na região central da cidade. Delegacias nas quais as mulheres que passam por situações de violência doméstica realizam os boletins de ocorrência no marco da Lei 11.340 de 2006 (Lei Maria da Penha) (Brasil et al., 2006).

Vítimas de violência doméstica são incentivadas para realizar a denúncia das agressões, no entanto, são poucas as mulheres, do total de vítimas, que a realiza. Na amostra analisada, somente 2 mulheres tinham denunciado. Entre os motivos que dificultam a denúncia das agressões ante a polícia têm sido pontoados: a baixa quantidade de policiais de sexo feminino, sentimentos de medo e vergonha por parte da denunciante, falta de acessibilidade dos serviços, crença de que a polícia não pode fazer nada a respeito, desejo de manter o incidente violento em privado, dificuldades de acesso à justiça e o baixo número de punições efetivas aos agressores (United Nations, 2015).

Estudos prévios no território brasileiro realizaram análise espacial de casos de violência doméstica sem achar padrões espaciais bem diferenciados. O que os autores do estudo atribuíram a que o fenômeno se distribui em todas as regiões do território atingindo todas as classes sociais. No entanto, chamam a atenção a que os riscos para a ocorrência do evento são maiores nos espaços geográficos de condições sociais menos favorecidas ou de maior desigualdade social (de Lucena et al., 2012), outros estudos levantaram o perfil dos feminicídios destacando caraterísticas como a raça branca, as baixa condição educativa e o perfil economicamente ativo das vítimas (de Santana et al., 2021). 
Mulheres vítimas de violência precisam de acesso a uma ampla gama de serviços prestados pela polícia, o sistema de justiça, e pelas redes e serviços de saúde e sociais que precisam estar coordenados para serem eficazes. Medidas específicas também são necessárias para facilitar que as mulheres deixem os relacionamentos violentos. Tais medidas devem levar em consideração o fato de que as mulheres são muitas vezes economicamente ou afetivamente dependentes dos parceiros íntimos e, portanto, correm o risco insegurança econômica o que dificulta o termino das relações violentas. Serviços de apoio especializado para mulheres, incluindo abrigos, ordens de proteção, aconselhamento e assistência jurídica, têm se mostrado eficazes em ajudar as mulheres a abandonar relacionamentos abusivos (United Nations Office on Drugs and Crime, 2019).

A legislação brasileira dispõe da Lei $N^{\circ} 13.104$ de 2015, mais conhecida como Lei de feminicídio (Brasil et al., 2015) que prevê o feminicídio como circunstância qualificadora do crime de homicídio e inclui o feminicídio no rol dos crimes hediondos com uma pena de reclusão de 12 a 30 anos. O crime é tipificado como feminicídio si é praticado contra a mulher por razoes da condição de sexo feminino quando envolve: violência doméstica e familiar, menosprezo ou discriminação à condição de mulher.

Mesmo que na atualidade mais países Latino-americanos tenham legislações especificas para o feminicídio como Argentina, Brasil, Colômbia, Costa Rica, Chile, Ecuador, El Salvador, Bolívia, Guatemala, Honduras, México, Nicarágua, Panamá, Peru, Venezuela e Republica dominicana (Essayag et al., 2017) as dificuldades persistem no continente, com altos níveis de agressões e assassinatos às mulheres. Devido às grandes dificuldades de acesso das vítimas a mecanismos jurídicos foi proposto o Protocolo Latino-americano para a investigação de assassinatos relacionados a gênero de mulheres (femicídio/feminicídio) (Sarmiento et al., 2014). Este protocolo foi adaptado para o contexto brasileiro mediante as Diretrizes Nacionais para investigar, processar e julgar com perspectiva de gênero as mortes violentas de mulheres (feminicídios) (Pasinato, 2016). Ditas diretrizes têm com o objetivo promover a inclusão de gênero na investigação criminal e no processo judicial em casos de mortes violentas de mulheres para seu correto enquadramento penal isenta de estereótipos e preconceitos de gênero para punir os responsáveis e garantir a reparação para vítimas e familiares.

No Brasil tem sido relatada uma experiencia de atenção com a criação de uma rede nacional de serviços integrais do tipo one stop (parada única) para acolher às vítimas e sobreviventes da violência. O programa "Mulher, viver sem violência" da Secretaria Especial de Políticas para as mulheres em 2015 inaugurou as "Casas da Mulher de Brasil” em Brasília e Campo Grande, como espaços de recepção e assistência humanizada que centralizam em um mesmo espaço físico os serviços especializados com una abordagem multidisciplinar (Essayag et al., 2017).

A violência e particularmente a violência letal é uma questão relevante de saúde pública em Campinas. A Fundação FEAC em 2019 destacou o homicídio doloso como um indicador social extremo de violência na cidade com uma taxa anual de 14 óbitos/100,000 hab. (FEAC, 2019). O que posiciona a Campinas acima da taxa média do estado. O principal grupo de vítimas são jovens (18-21 anos e 25-29 anos) de sexo masculino. As ocorrências acontecem principalmente dentro ou próximas a áreas de vulnerabilidade social. A respeito dos homicídios femininos estimou-se que a taxa e sete vezes inferior quando comparada ao sexo masculino, contribuindo aproximadamente com um 8\% do total de casos (FEAC, 2019).

Padrões de homicídio de homens e mulheres são diferentes. Os homicídios masculinos acontecem notadamente no período da noite à madrugada nas vias públicas, de forma rápida e abrupta e em locais que permitem a evasão dos responsáveis (FEAC, 2019), por sua parte, os feminicídios são notadamente residenciais, perpetrados por pessoas conhecidas da vítima (namorado, conjugue, filho, neto, ex-parceiro) e mediante o uso de objetos perfurocortantes.

A fundação FEAC tinha identificado sete Hotspot o clusters de maior ocorrência de casos de homicídio doloso em Campinas. Um deles na região Sudeste, caracterizada por ser uma região com alta densidade populacional, e dois na região noroeste, um em uma parcela de território isolado de grandes áreas de campos abertos e outro em uma área de vulnerabilidade social com empreendimentos de moradia popular (FEAC, 2019). O que indica que estas regiões presentam um diferencial social 
em termos de vulnerabilidade e violência e seus moradores estão expostos a dinâmicas de violência mais intensas e perigosas que moradores de outros setores na cidade.

Diante de casos de feminicídio é possível identificar a falência nos mecanismos da cidade para eliminar a violência contra a mulher. Estratégias devem integrar quatro eixos de intervenção: prevenção, atenção, sanção e reparação (Essayag et al., 2017). Ações na América Latina e no Brasil especificamente estão voltadas sobre os três primeiros eixos, mas ante os casos de feminicídio é fundamental a reparação das vítimas e das sobreviventes.

Ações no eixo da prevenção incluem: campanhas educativas, de sensibilização, capacitação e conscientização; criação de ambientes de tolerância cero e ambientes institucionais seguros; articulação de redes para a discussão com crianças, jovens e adultos sobre masculinidades não violentas; políticas públicas que favorecem a proteção das mulheres e limitam o uso de elementos que podem aumentar as violências (armas, consumo de álcool e drogas). Ações no eixo da atenção estão voltadas sobre a criação de espaços seguros como refúgios e casas abrigo; articulação multidisciplinar para a atenção de vítimas de violência com atenção médica, psicológica e jurídica no mesmo espaço; protocolos de atenção; linhas de comunicação, assessoramento e denúncia. Ações no eixo da sanção incorporam instrumentos legislativos e de políticas públicas para garantir a proteção das vítimas, aumentar as penas dos agressores e leis para erradicar condutas sexistas; criação de policias especializadas para responder a violência e operadores de justiça e programas de reeducação de agressores. Finalmente as ações de reparação são aquelas encaminhadas para a reparação do dano moral e reparação à violação dos direitos das vítimas e sobreviventes como a assistência psicológica, jurídica, social e econômica, acesso a moradia, medicamentos, insumos e tratamentos necessários, mecanismos de reintegração a programas de emprego e formação e substituição de bens destruídos ou compensação econômica (Essayag et al., 2017).

\section{Considerações sobre a análise espacial}

A aplicação de métodos de análise espacial é realizada a partir da integração de pontos espaciais únicos $(x, y)$ de latitude e longitude, ou por meio de áreas definidas que compartilham características em polígonos, que representam as unidades de análise. O sistema de coordenadas latitude e longitude permite expressar uma posição geográfica de qualquer lugar no mundo e é a escolha metodológica mais custo-efetiva (Pfeiffer D et al., 2008). As análises se correspondem com representações em escala da realidade no território e requerem ajustes matemáticos para representar dados que vem de um globo em uma superfície plana.

Sabe-se que as regiões vizinhas tendem a apresentar similitudes em comparação com as regiões más distantes (Morgenstern, 2008). Assim, dados coletados de pontos mais próximos são mais similares quando comparado com outros de pontos mais distantes. O que se conhece como dependência espacial (Pfeiffer D et al., 2008). Que os dados espaciais sejam dependentes viola o princípio estatístico clássico de independência. A consequência dessa violação na análise é a subestimação dos erros e a superestimação da significância estatística incrementando consequentemente o erro tipo I. diferentes aproximações têm sido usadas para lidar com esta questão. Modelar a dependência como parte da variância é o método implementado no modelo linear generalizado (Pfeiffer D et al., 2008).

Uma consideração de interesse para a análise espacial é o efeito de borda. As bordas são resultado de barreiras físicas como rios, mares, fronteiras, divisões administrativas ou outros que definem as bordas dos perímetros estudados. É usual que desses dados estejam incompletos ou não disponíveis, pelo que os dados coletados nesses lugares vão ter menos vizinhos que os pontos coletados no centro da área de estudo. A presença de poucos dados pode gerar distorção nas estimativas das áreas perto das bordas, o que se conhece como efeito de borda (Pfeiffer D et al., 2008). 


\section{Considerações Finais}

Tanto na análise espacial quanto nos modelos de análise da violência se reconhece a confluência de fatores ambientais, culturais, relacionais, individuais e geográficos para que aconteçam os casos. A analise espacial é uma estratégia complementar que parte das características particulares dos casos para elaborar uma compreensão mais ampla do fenômeno da violência contra a mulher, particularmente na análise de casos de feminicídio integrar esta análise permite agregar diferentes áreas do conhecimento como a saúde pública, a epidemiologia, os estúdios de analise espacial e os conceitos de poder provenientes dos estudos de gênero.

São poucos os trabalhos em Campinas que têm analisado o fenômeno dos feminicídios na cidade, assim este trabalho é o primeiro que realiza uma análise espacial partindo de um modelo de casos e controles que permitiu identificar as regiões noroeste e sudoeste como as de mais risco na cidade para feminicídio. A identificação de regiões de risco é de utilidade para aplicar intervenções focadas nas caraterísticas dos territórios e subsidiar a tomada de decisões dos gestores. Para pesquisas futuras na área de violência letal e analise espacial seria interessante determinar as áreas de abrangência das delegacias na cidade, isso significa, identificar se as denunciantes provem das regiões marcadas com maior risco de morte por feminicídio. É provável que estas regiões estejam subrepresentadas e sejam necessárias medidas locais especificas ou a localização de uma terceira delegacia mais perto da área de risco para facilitar o acesso às mulheres em condição de violência.

Que existam casos de feminicídio em Campinas é reflexo do limitado impacto das ações para prevenir, erradica e punir as violências contra as mulheres. Enfoques voltados sobre os eixos de prevenção, atenção, sanção e reparação são necessários para garantir para meninas, jovens e mulheres do território entornos saudáveis e seguros de ações violentas e potencialmente mortais.

\section{Fonte de financiamento}

O presente artigo faz parte da produção acadêmica da tese de doutorado intitulada "Fatores de risco para feminicídios na cidade de Campinas, revisão da literatura, estudo caso-controle espacial e análise qualitativa" do programa de Saúde Coletiva da Faculdade de Ciências Médicas da Universidade Estadual de Campinas- Unicamp financiado mediante uma bolsa de estudos outorgada pela Coordenação de Aperfeiçoamento de Pessoal de Nível Superior (Capes) código 001.

\section{Referências}

Antipon, L. C. (2018). Um estudo da história territorial de Campinas: a urbanização, a fome e a formação de um mercado de alimentação na cidade (1850-1908). Revista da Associação Nacional de Pós-graduação e Pesquisa em Geografia (Anpege), 14(23): 28-58.

Lei N 11.340 , de 7 de agosto de 2006, (2006). http://www.planalto.gov.br/ccivil_03/_ato2004-2006/2006/lei/111340.htm

Lei $\quad N^{\text {o }} \quad 13.104, \quad$ de 9 de Março de 2015, (2015). http://www.planalto.gov.br/ccivil_03/_ato20152018/2015/lei/113104.htm\#: :text=Altera\%20o\%20art.,no\%20rol\%20dos\%20crimes\%20hediondos

Caicedo-Roa, M., do Nascimento, J., Bandeira, L., \& Cordeiro, R. (2021). Queima às bruxas: Feminismo e Feminicídios íntimos por queimadura em uma metrópole. Cien Saude Colet. https://www.cienciaesaudecoletiva.com.br/artigos/queima-as-bruxas-feminismo-e-feminicidios-intimos-por-queimadura-emuma-metropole/17933?id=17933

Caicedo-Roa, M., Cordeiro, R., Martins, A., \& Faria, P. (2019). [Femicides in the city of Campinas, Sao Paulo, Brazil] [Femicidios na cidade de Campinas, Sao Paulo, Brasil. DEP - 20190704]. Cad. saúde pública., (1678-4464 (Electronic)).

Campbell, J. C., Glass, N., Sharps, P. W., Laughon, K., \& Bloom, T. (2007). Intimate partner homicide: Review and implications of research and policy. Trauma Violence Abuse, 8(3): 246-269.

Cano, W., \& Brandão, C. A. (2002). A região metropolitana de Campinas: urbanização, economia, finanças e meio ambiente. Editora Unicamp.

Carvalho, M. S., \& Souza-Santos, R. (2005). Análise de dados espaciais em saúde pública: métodos, problemas, perspectivas. Cad. saúde pública. 21: 361-378.

Cerqueira, D., \& Bueno, S. C. (2019). Atlas da Violência 2019. In Instituto de Pesquisa Econômica Aplicada (Ipea) \& Fórum Brasileiro de Segurança Pública (FBSP) (Eds.): Brasília. 
Cordeiro, R. (2018). A inadequação da classificação oficial dos acidentes de trabalho no Brasil. Cad. saúde pública., 34, e00173016.

Cordeiro, R. (2018). Trabalho, violência e morte: miséria da existência humana. Appris.

Cordeiro, R., Luz, V. G., Hennington, É. A., Martins, A. C. A., \& Tófoli, L. F. (2017). A violência urbana é a maior causa de acidente de trabalho fatal no Brasil. Rev. Saúde Públ., 51, 123.

Câmara Municipal de Campinas. (2015). Conheça Campinas. http://www.campinas.sp.leg.br/institucional/conheca-campinas

de Lucena, K., da Silva, A. T., de Moraes, R. M., da Silva, C. C., \& Bezerra, I. (2012). Análise espacial da violência doméstica contra a mulher entre os anos de 2002 e 2005 em João Pessoa, Paraíba, Brasil. Cad. saúde pública [online], 28(6). https://doi.org/10.1590/S0102-311X2012000600010

de Santana, A. C. C. S., dos Santos, L. S., de Jesus Guimarães, J., de Carvalho Barreto, I. D., Lima, S. O., de Melo, C. M., \& Reis, F. P. (2021). Perfil do feminicídio: Uma abordagem epidemiológica no Estado de Sergipe. Res., Soc. Dev, 10(5): e47310515197.

Ellsberg, M., Peña, R., Herrera, A., Liljestrand, J., \& Winkvist, A. (2000). Candies in hell: women's experiences of violence in Nicaragua. Soc Sci Med, 51(11): 1595-610.

Essayag, S., PNUD, \& ONU Mujeres. (2017). Del Compromiso a la Acción: Políticas para erradicar la violencia contra las mujeres en América Latina y el Caribe, 2016. Panamá.

FEAC. (2019). Mapa da violência de Campinas-Diagnóstico Socioterritorial.

Hennington, É. A., Cordeiro, R., \& Moreira Filho, D. d. C. (2004). Trabalho, violência e morte em Campinas, São Paulo, Brasil. Cad. saúde pública., 20: 610617.

Instituto Brasileiro de Geografia e Estatística. (2021a). Estimativas de população publicadas no DOU. Tabelas de estimativas populacionais para os municípios e para as Unidades da Federação brasileiros. https://www.ibge.gov.br/estatisticas/sociais/populacao/9103-estimativas-de-populacao.html?=\&t=resultados

Instituto Brasileiro de Geografia e Estatística. (2021b). IBGE divulga estimativa da população dos municípios para 2021. https://agenciadenoticias.ibge.gov.br/agencia-sala-de-imprensa/2013-agencia-de-noticias/releases/31461-ibge-divulga-estimativa-da-populacao-dosmunicipios-para-2021

Instituto Patricia Galvão. (2019). Dossiê Feminicídio. O que é Feminicídio? https://dossies.agenciapatriciagalvao.org.br/feminicidio/capitulos/o-que-efeminicidio/

Lora, A. P. (2010). Distribuição espacial do risco de hipertensão arterial sistêmica e diabetes mellitus em área do Municipio de Pedreira. (Publication Number CRB 8 / 6652) Universidade Estadual de Campinas]. Campinas.

Meneghel, S. N., \& Hirakata, V. N. (2011). Femicídios: homicídios femininos no Brasil. Rev. Saúde Públ., 45: 564-574.

Morgenstern, H. (2008). Ecologic studies. In K. Rothman (Ed.), Modern epidemiology (3a ed.), pp. 511-531. Lippincott Williams \& Wilkins.

Núcleo de Gênero Ministerio Público do Estado de São Paulo. (2018). Raio X do feminicídio em São Paulo É possível evitar a morte. http://www.compromissoeatitude.org.br/raio-x-do-feminicidio-em-sao-paulo-promotora-valeria-scarance-reforca-que-e-possivel-evitar-morte/

Pasinato, W. (2016). Diretrizes Nacionais Feminicídio: investigar, processar e julgar com perspectiva de gênero as mortes violentas de mulheres.

Pfeiffer D, Robinson T, Stevenson M, Stevens K, Rogers D, \& Clements A. (2008). Spatial Analysis in Epidemiology. In: Oxford Scholarship Online.

Programa das Nações Unidas para o Desenvolvimento. (2020). O que é o IDHM. https://www.br.undp.org/content/brazil/pt/home/idh0/conceitos/o-que-e-oidhm.html

R Core Team. (2014). R: A language and environment for statistical computing. R Foundation for Statistical Computing. http://www.R-project.org/

Rizzatti, H. (2015). As maiores ocupações urbanas da cidade de Campinas-SP: resistências e permanência. Revista de Ciências Humanas, 49(2): 183-204.

Sarmiento, C. B., Acosta, M. L., Roth, F., \& Zambrano, M. (2014). Latin American model protocol for the investigation of gender-related killings of women (femicide/feminicide). United Nations.

Shimakura, S. E., Carvalho, M. S., Aerts, D. R. G. C., \& Flores, R. (2001). Distribuição espacial do risco: modelagem da mortalidade infantil em Porto Alegre, Rio Grande do Sul, Brasil. Cad. saúde pública., 17: 1251-61.

Sosa, L. P. A. (2017). Inter-American case law on femicide: Obscuring intersections? Netherlands Quarterly of Human Rights, 35 (2): 85-103.

StataCorp. (2011). Stata Statistical Software: Release 12 . College Station, TX: StataCorp LP. https://www.stata.com/

Stephan, C. (2008). Distribuição espacial do risco do acidente do trabalho entre trabalhadores precarizados de Piracicaba Universidade Estadual de Campinas]. Campinas, SP.

Unicamp, \& Faculdade de Ciências Médicas. (2020). Linhas de pesquisa. Área de concentração: Epidemiologia. https://www.fcm.unicamp.br/fcm/posgraduacao-em-saude-coletiva/linhas-de-pesquisa

United Nations. (2015). The world's women 2015: trends and statistics. New York (NY): United Nations, Department of Economic and Social Affairs. Statistics Division.

United Nations, \& Economic and Social Council. (2013). Vienna Declaration on Femicide. Viena. 
Research, Society and Development, v. 11, n. 1, e17811125011, 2022

(CC BY 4.0) | ISSN 2525-3409 | DOI: http://dx.doi.org/10.33448/rsd-v11i1.25011

United Nations Office on Drugs and Crime. (2019). Global Study on Homicide: Gender-related Killing of Women and Girls. UNODC, United Nations Office on Drugs and Crime. https://www.unodc.org/unodc/en/data-and-analysis/global-study-on-homicide.html

World Health Organization. (2007). Verbal autopsy standards: ascertaining and attributing cause of death. World Health Organization.

World Health Organization. (2013). Global and regional estimates of violence against women: prevalence and health effects of intimate partner violence and non-partner sexual violence. World Health Organization. 